\title{
Novel adaptation of the spectral kurtosis for vibration diagnosis of gearboxes in non-stationary conditions
}

\author{
L Gelman, S Kolbe, B Shaw and M Vaidhianathasamy
}

\begin{abstract}
In this paper, the adaptation of spectral kurtosis technology is proposed, demonstrated and experimentally validated. Raw data signals were collected from a single-stage gearbox run in different combinations of speed and load, after which time synchronous averaging was used to leave the classical residual signal once meshing harmonics were removed. Each data file is split into many individual realisations based on the time taken for the time synchronous average to converge on stable values, after which the short-time Fourier transform is used to calculate the spectral kurtosis for each realisation. The effects of adapting spectral kurtosis technology parameters such as the resolution and threshold used in creating a Wiener filter are evaluated, showing the effects on the consistent frequency bands identified throughout the realisations. Taking a baseline set of processing parameters, the probability of correct diagnosis was calculated using a three-stage decision-making technique incorporating the k-nearest neighbour and cluster analysis methods. Adaptation of the spectral kurtosis technology is then shown to dramatically improve the probability of correct diagnosis, highlighting that each speed and load case requires different resolution and threshold values to return the
\end{abstract} optimal results.

\section{Introduction}

Condition monitoring of rotating machinery is an ever-evolving area of research, with a large amount focusing on vibrational signal analysis by employing time-frequency transforms and spectral analysis. However, most of this research is based on data collected from machinery run in constant speed and load conditions, thereby increasing the chances of a correct machinery condition diagnosis. In such a situation, the only variable that changes between datasets is the presence of damage, meaning algorithms and signal processing can be extremely sensitive to such fluctuations.

The bulk of research into vibrational analysis of rotating machinery can be traced back to the initial proposal of time synchronous averaging (TSA) by McFadden ${ }^{[1,2]}$. More specifically, the application of TSA to gearbox teeth and meshing components was first approached by Badaoui et al ${ }^{[3]}$, whereby complex FEA models were built to theoretically demonstrate and compare various diagnostic techniques already proposed in ${ }^{[1,2]}$. Practical proof of the methods was performed by Bonnardot et al ${ }^{[4]}$ before developing the concept of angular resampling of signals to obtain instantaneous shaft positions. This was further developed by Combet and Gelman ${ }^{[5]}$ to allow for speed estimation without a tachometer, meaning that gearbox operating conditions could be accurately observed at all times.

In the field of bearing and gear diagnosis, it was observed that the spectral kurtosis (SK) is sensitive to non-stationary changes in a vibrational signal and as a result is able to indicate the frequencies at which these non-stationary signal components occur ${ }^{[6,7,8]}$. The SK-derived Wiener filter was born from this field after it was observed that the SK and a Wiener filter (widely accepted as being an optimal denoising filter) had a very similar mathematical composition and could be applied to dramatically enhance small transients in vibration signals caused by tooth damage ${ }^{[8]}$. This has now become standard filtering in the signal processing field.

There are several methods that can be used to validate the results of new research. The Fisher criterion indicates the effectiveness of a change in processing parameters or algorithms and allows a comparison between multiple sets of results ${ }^{[8,9]}$. An alternative method is to use automated decision-making codes to perform damage diagnosis and return a 'probability of correct diagnosis', which is a more useful measure of improvement between processing attempts $^{[10,11]}$.

It is widely accepted that damage diagnosis in gearboxes operated in non-stationary conditions is particularly challenging due to the fact that vibrational diagnostic features are speed and load dependent ${ }^{[12]}$. Ongoing research in the area primarily concentrates on identifying the changes to the raw signal as well as attempting to find new damage indicators ${ }^{[12,13,14]}$. The analysis focuses on trying to find new methods to detect damage, rather than adapting known techniques to match the varying operating conditions. Adaptive methods have been used to more accurately detect the gearbox state based on load but not to diagnose damage ${ }^{[15]}$. The adaptation of wavelet and classical residual technology for gearboxes operated with varying speed and load was proposed by Gelman and presented at the International Condition Monitoring Conference in 2012 $2^{[16]}$ However, based on a comprehensive literature review, the authors believe that nobody has yet proposed, investigated and validated adaptation of the SK technology to the varying speed and load of

- Based on a paper presented at CM 2016/MFPT 2016, the Thirteenth International Conference on Condition Monitoring and Machinery Failure Prevention Technologies, Paris, France, October 2016. This paper was a winner of the Len Gelman Award for the best paper in the proceedings by a person in the early stages of their career.

L Gelman and S Kolbe are with Cranfield University, Cranfield, Bedfordshire MK43 OAL, UK.

B Shaw and M Vaidhianathasamy are with Newcastle University, Newcastle upon Tyne NE1 TRU, UK. 
gearboxes.

This paper begins to explore the effects of varying speed and load conditions on the signal responses of gearbox vibrational data. Furthermore, an adaptation of SK technology that increases the probability of correct diagnosis for variable speed and load conditions is proposed here.

The main objectives of this paper are:

- To propose, develop and experimentally validate a novel adaptation of the SK technology for gearboxes in non-stationary conditions of speed and load;

- To compare the consistent frequency bands identified across different combinations of speed and load for both damaged and undamaged cases;

- To show that the optimal parameters to use when adapting the SK technology vary depending on operating conditions;

- To compare the adaptive and non-adaptive technologies to demonstrate the gains achievable in the total probability of error diagnosis.

The result of this is to allow the SK technology to be more effectively applied to real-world scenarios where the machinery being monitored is rarely run in one set of conditions for its entire lifetime. The overall aim is to improve the effectiveness of diagnosis of a gearbox regardless of the operating parameters by adapting the SK technology. Speed and load channels/sensors could be utilised to allow for online adaptation of the automated processing and decision making.

\section{The spectral kurtosis}

The spectral kurtosis is a measure of the 'peakedness' of a frequency distribution, more accurately described as the deviation from Gaussianity, and has long been used as a signal processing tool for advanced damage detection diagnosis of rotating machinery, particularly bearings and gears. This is due to its inherent sensitivity to non-stationary changes in a signal and is thus capable of detecting the frequencies at which these changes occur ${ }^{[7,6,8]}$.

The spectral kurtosis of a signal is defined as the fourth-order spectral moment, given through:

$$
K=n \frac{\sum_{i=1}^{n}\left(x_{i}-x_{\text {avg }}\right)^{4}}{\left[\sum_{i=1}^{n}\left(x_{i}-x_{\text {avg }}\right)^{2}\right]^{2}}
$$

where $K$ is spectral kurtosis, $x_{i}$ and $x_{\text {avg }}$ represent each individual data point and the average of all data points, respectively. If this is performed in the time domain, it is simply the kurtosis.

Another advantage is the similarity between the spectral kurtosis and the Wiener filter, which is commonly accepted as one of the optimal filtering technologies within the signal processing field. The two are linked through $W(f) \propto \sqrt{K_{x}(f)}$, so the Wiener filter is created by identifying where the SK value is greater than a preset threshold (otherwise $W(f)=0$ ). As such, the SK-based Wiener filter has become standard in damage diagnosis research in order to isolate changes to the signal's frequency spectrum due to damage ${ }^{[8]}$.

Once a vibrational signal has been obtained in the time domain, it is divided into individual realisations, the length of which is based on the number of shaft periods taken for the TSA response to converge on a stable value ${ }^{[5]}$. Once the TSA has been calculated for each realisation and harmonics at meshing frequencies removed, the result is a set of classical residual signals ${ }^{[1]}$.

Utilising the short-time Fourier transform (STFT), the classical residual signals are converted into the frequency domain, whereby an overlapping window (for example a Hanning window) is applied to effectively smooth the response before the SK at each frequency is calculated from the magnitude of the STFT across a window of pre-defined length:

$$
K_{x}(f)=\frac{S_{4, x}(f)}{S_{2, x}(f)}-2
$$

where $S_{n, x}(f)=\left\langle|X(t, f)|^{n}\right\rangle$ and is the complex envelope of signal $x(t)$, which can be estimated by the STFT.

The length of the smoothing (ie length of window) is described as the SK time resolution and has a large impact on the overall results and separation of the signal between damaged and undamaged cases.

Wherever the calculated SK is above a specified threshold, the SK-based Wiener filter is derived and can then be applied back to the original signal in order to formulate an SK residual. It is from this that a diagnostic feature (typically a squared energy envelope) is calculated prior to automated decision-making based on cluster analysis and $k$-nearest neighbours.

Effective selection of the SK resolution and SK threshold have a significant impact on the effectiveness of the derived Wiener filter and, therefore, these technology parameters are among those which should be optimised/adapted based on the dataset being considered.

\section{The consistency parameter of the SK}

The consistency parameter has been proposed by Gelman ${ }^{[17]}$ as a methodology for identifying a frequency band that has a consistently high peak across the majority of realisations in an analysed data file. This peak would form the basis of the SK-derived Wiener filter. That work was limited to the identification and calculation of consistency when applied to data containing only one significant peak in the spectral kurtosis and assuming that any peak was likely to be at the same frequency in all the realisations. The only check for this was a visual one by the operator. It was also noted that if the SK threshold is incorrectly chosen, this will potentially lead to the SK being zero across an entire realisation and, as thus, no peak will be detected.

By continuing to develop the algorithm, it has been possible to add the capability to allow for the identification of multiple consistent frequency bands across the realisations in a dataset and independently calculate their consistency parameter.

To calculate the consistency parameter(s), spectral kurtosis values are assigned either a 0 or 1 based on whether they fall above or below the selected SK threshold. By performing equal width binning on the SK data, each bin for each realisation is then assigned a 0 or 1 depending on whether there is a non-zero SK value within that frequency range. The consistency parameter is then evaluated per bin by evaluating the formula below:

$$
p_{b}=\frac{\sum C M_{b}}{\text { Nreal }} \times 100
$$

where $p$ is the consistency parameter (in percent), $C M$ is the consistency matrix for each bin, Nreal represents the total number of realisations and subscript $b$ denotes the bin number.

The newly-developed algorithm then performs a second iteration of the same calculation, in which the bin widths are automatically adjusted so that if neighbouring bins both show high consistency parameter values they are merged into one bin for the second iteration. This removes any calculation errors that may come from SK peaks spread across two of the initial bins.

The outcome of this process is to identify frequency bands that 
have consistently high SK values across all realisations in a data file and which cannot be due to noise or other discrepancies in the raw data. Ensuring that the SK threshold is chosen to still allow for some highly-consistent frequency bands results in a higher likelihood of correct diagnosis, as the vibration signature across all realisations is comparable and the resulting SK-based Wiener filter is more likely to isolate diagnostic features rather than noise.

\section{Adaptation of the SK technology}

The research presented in this paper is focused on applying an advanced vibrational signal processing technique to data collected from gearboxes being run in varying speed and load conditions. Typical data capture is from rotating machinery running at a constant speed and load for its entire lifetime, which is not representative of actual operational conditions outside of research. Identical processing parameters are then applied across datasets and often these parameters are not optimised; merely trial and error finds values that perform adequately. In this scenario, the presence of damage is the only system change between cases and therefore the system can be accurately tuned to give high diagnosis probabilities or, conversely, an adequate result can be achieved with sub-optimal parameters.

Many real-world systems (cars/aircraft jet engines and gearboxes, industrial motors and gearboxes, wind turbines, etc) have several sets of standard operating conditions of speed and torque, for example:

- Low speed, low load: for example idling

- Low speed, high load: for example acceleration from stationary

- High speed, low load: for example cruising once the desired speed is achieved

- High speed, high load: for example acceleration when already in motion.

Therefore, the main novel aspect of this research is to propose, investigate and validate that the SK technology should be adapted to the varying speed and load conditions of gearboxes in order to increase the effectiveness of diagnosis. The gearbox condition must be effectively diagnosed in all operational states for successful condition monitoring as, if damage is only detected in one set of running parameters, it may be many hours between damage occurring and it being detected.

With the proposed adaptation, the SK-derived Wiener filter will be composed only of frequency bands that have a high consistency across all realisations, meaning the resulting probability of diagnosis will be increased. The consistent frequency bands identified are likely to be different for each combination of running parameters, along with the SK technology parameters that best identify them and subsequently lead to the highest probability of correct diagnosis.

This paper shows that adapting the main SK technology parameters (the SK resolution (SKres) and the SK threshold (SKthres)) results in changes to the consistent frequency bands detected for each set of operating conditions and that fully adapting SK parameters to varying speed and load can significantly improve the probability of correct damage diagnosis.

This adaptation has been applied to both datasets with and without damage, obtained from a back-to-back gearbox test set-up run in four combinations of speed and load. Any changes in consistent frequency bands across the datasets were then investigated. The results of this study highlight the need to alter technology parameters to the operating conditions in order to achieve optimal diagnosis outcomes in all circumstances.

\section{Experimental results}

As detailed in Section 2, the general process followed is widely accepted in the field as being standard for pre-processing of a vibrational signal. The time synchronous averaging is performed, in this case using a tacho pulse to index each rotation, to eliminate interference and non-stationary components from the signal. After removal of meshing frequencies, the result is the classical residual signal, which is taken forwards for SK-based filtering. A typical data file in this analysis resulted in over 150 realisations, each $8 \mathrm{~s}$ long as this is enough time to ensure convergence of the residual signal in all speed conditions.

The SK resolution value is an input to the software, which affects both the smoothing of the signal and the window length for calculation of the SK. The SK is then calculated using the STFT technique and, after setting an SK threshold value, the consistent frequency bands for each speed and load combination are observed.

The remainder of the paper will focus on two combinations of running parameters. These speed and load conditions contain one at low speed and low load ( $1500 \mathrm{r} / \mathrm{min}, 250 \mathrm{Nm})$ and the other at high speed and high load (3000 r/min, $500 \mathrm{Nm}$ ).

The gearbox used for acquiring data was a back-to-back set-up at the Gear Research Centre within Newcastle University and was operated by B Shaw and M Vaidhianathasamy, who collected the data but had no involvement in the signal processing or condition monitoring optimisation. The set-up contained two gearboxes, each with a ratio of 1:1.5 (ie 16 teeth on the pinion and 24 teeth on the wheel) connected by two torsionally-compliant shafts, one of which was connected to the motor and the other to a torque actuator used to load the gearboxes.

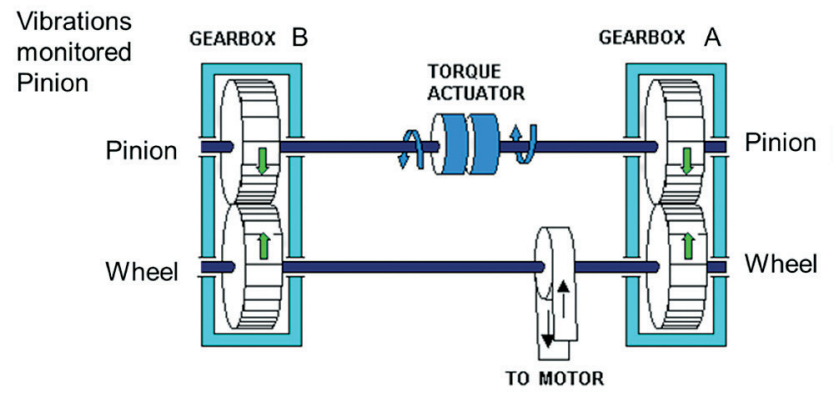

Figure 1. Illustration of the test-rig set-up

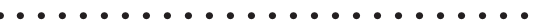

During testing, there were both optical and proximity speed sensors placed on the input shaft as well as two tri-axial accelerometers on the gearbox housing. All data was logged at $40 \mathrm{kHz}$ using a LabView program to store the data as .bin files for processing.

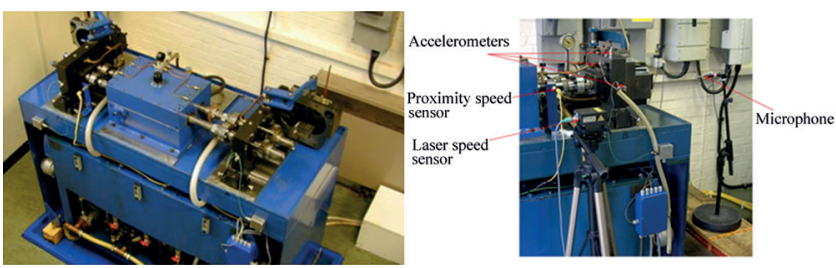

Figure 2. Experimental gearbox set-up

\subsection{Non-adapted SK processing}

As a baseline, the data files were processed using identical SK parameters, which allows the impact of changing the SK technology variables to then be demonstrated. 
The data represented in Figures 3 and 4 represents the low-speed, low-load data and high-speed, high-load data, respectively. At this stage the data files were processed with a baseline set of values, ie SK resolution of mesh period and SK threshold of 0.2 , which from experience are predicted to give adequate results.

It can be clearly seen that there are consistent frequency bands visible on both the undamaged and damaged data; however, the number of consistent bands and the frequencies at which these occur are different (Figures 3(c) and 3(d)). There is not a large change in consistency parameter value between the undamaged and damaged cases.

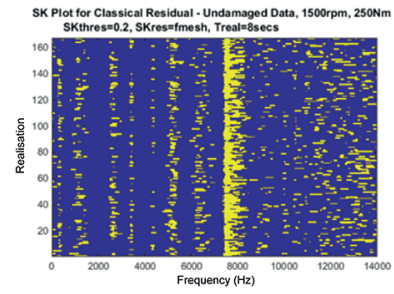

(a)

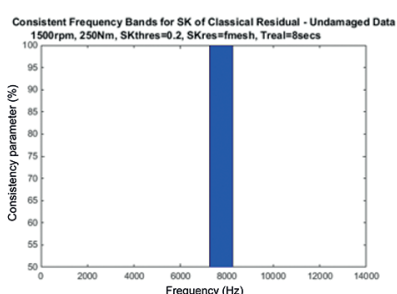

(c)

\begin{tabular}{|c|c|}
\hline Central Freq $(\mathrm{Hz})$ & Consistency $(\%)$ \\
\hline 7711 & 100 \\
\hline
\end{tabular}

(e)

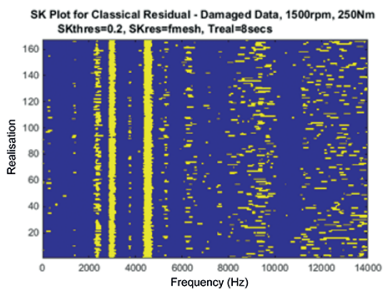

(b)

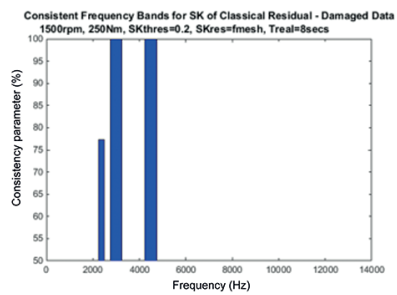

(d)

\begin{tabular}{|c|c|}
\hline Central Freq (Hz) & Consistency (\%) \\
\hline 2369 & 77.2 \\
\hline 2993 & 100 \\
\hline 4489 & 100 \\
\hline
\end{tabular}

(f)
Figure 3. SK plots and consistent frequency bands for gearbox data collected at $1500 \mathrm{r} / \mathrm{min}$ and $250 \mathrm{Nm}$. SK resolution is fmesh, SK threshold is 0.2 and a realisation time of $8 \mathrm{~s}$ has been used throughout: (a) SK plot of classical residual - undamaged; (b) SK plot of classical residual - damaged; (c) consistent frequencies undamaged; (d) consistent frequencies - damaged; (e) consistent frequencies - undamaged; (f) consistent frequencies - damaged

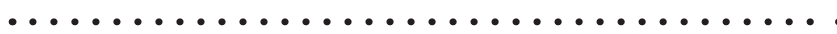

The areas of high consistency in the undamaged data is an indication of signal elements unrelated to the gear meshing but that are affecting the recorded vibration signal. As the diagnostic feature is unaffected, it appears that these are independent of gear meshing. The adaptation techniques explored later in the paper demonstrate that even with these artefacts in the signal, effective correct diagnosis is still achievable.

The high-speed and high-load data (Figures 4(c) and 4(d)) exhibits the same number of consistent frequency bands when compared to Figure 3, yet only one of them is similar between the two speed and load cases. In all cases the consistency is high.

\subsection{Adaptation of the SK}

The data files were processed again while simultaneously adapting both the SK resolution and the SK threshold to find the best possible probability of correct diagnosis for each speed and load case. The results can be seen in Figures 5 and 6 .

This set of adapted parameters (the optimal combination of all those tested) has the effect of completely removing any consistent frequency bands for the undamaged data and leaving only one

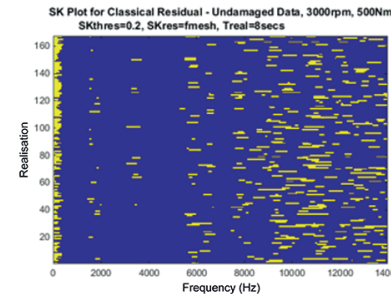

(a)

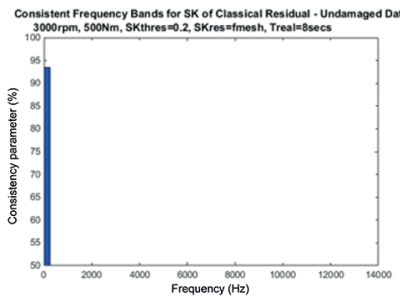

(c)

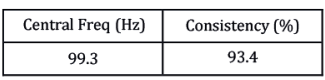

(e)

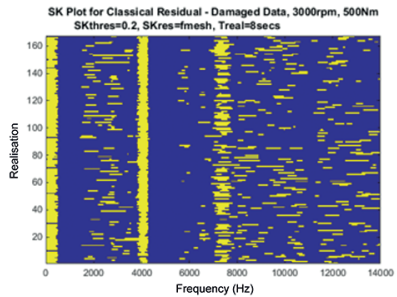

(b)

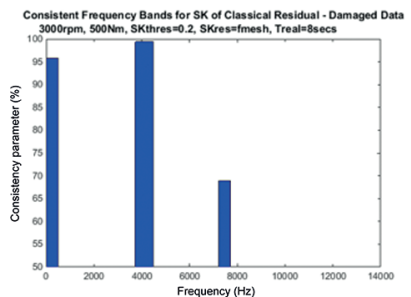

(d)

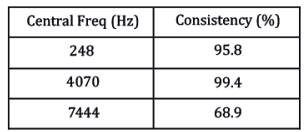

(f)
Figure 4. SK plots and consistent frequency bands for gearbox data collected at $3000 \mathrm{r} / \mathrm{min}$ and $500 \mathrm{Nm}$. SK resolution is fmesh, SK threshold is 0.2 with a realisation length of $8 \mathrm{~s}$ : (a) SK plot of classical residual - undamaged; (b) SK plot of classical residual damaged; (c) consistent frequencies - undamaged; (d) consistent frequencies - damaged; (e) consistent frequencies - undamaged; (f) consistent frequencies - damaged

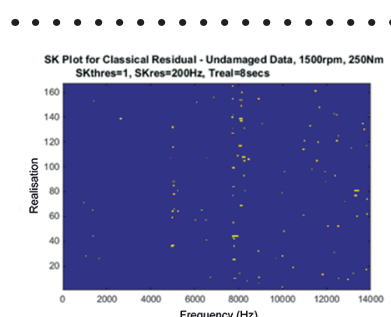

(a)

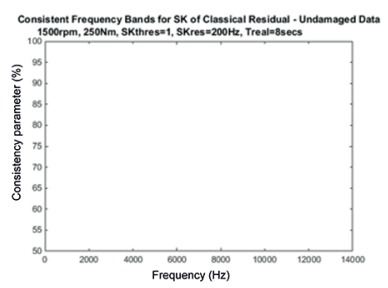

(c)

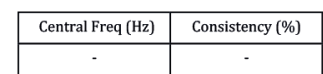

(e)

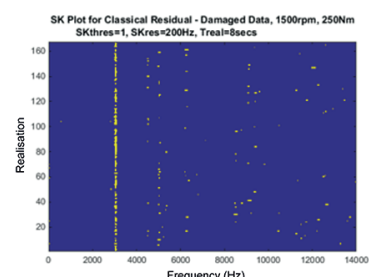

(b)

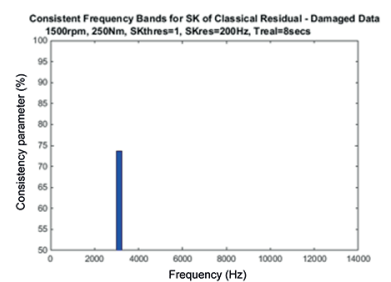

(d) (f)
Figure 5. SK plots and consistent frequency bands for gearbox data collected at $1500 \mathrm{r} / \mathrm{min}$ and $250 \mathrm{Nm}$, when processed with an SK resolution of $200 \mathrm{~Hz}$ and SK threshold of 1: (a) SK plot of classical residual - undamaged; (b) SK plot of classical residual damaged; (c) consistent frequencies - undamaged; (d) consistent frequencies - damaged; (e) consistent frequencies - undamaged; (f) consistent frequencies - damaged

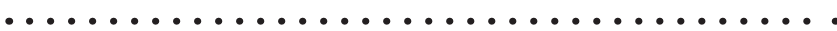

relatively consistent peak in the damaged data. This is likely to result in a high probability of correct diagnosis, which testing more combinations of parameters may increase further if it is possible to increase the consistency of the damaged data. 


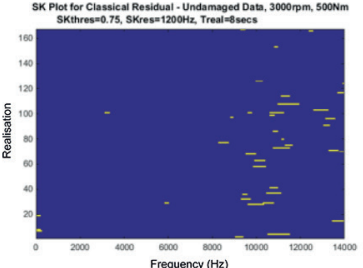

(a)

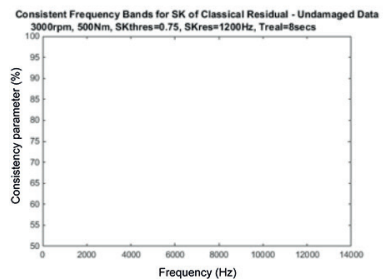

(c)

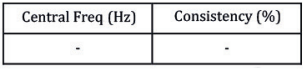

(e)

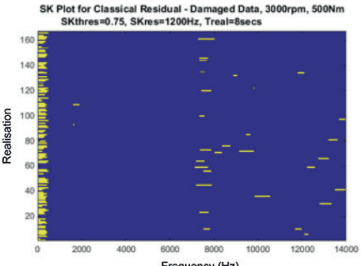

(b)

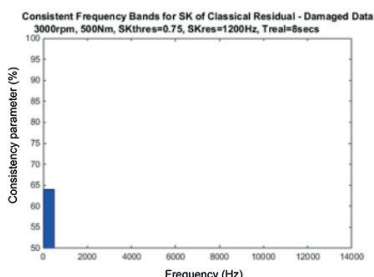

(d)

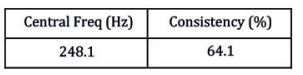

(f)
Figure 6. SK plots and consistent frequency bands for gearbox data collected at $3000 \mathrm{r} / \mathrm{min}$ and $500 \mathrm{Nm}$, when processed with an SK resolution of $1200 \mathrm{~Hz}$ and SK threshold of 0.75: (a) SK plot of classical residual - undamaged; (b) SK plot of classical residual damaged; (c) consistent frequencies - undamaged; (d) consistent frequencies - damaged; (e) consistent frequencies - undamaged; (f) consistent frequencies - damaged

Again, the adaptation has completely removed any consistent frequency bands from the undamaged data and isolated just one in the damaged data. As with the low speed and load data seen previously, by testing even more combinations of speed and load it may be possible to further improve this one consistent frequency band and ultimately improve the diagnosis probability.

Whilst the outcomes of the SK adaptation are similar (removing all consistent bands from the undamaged data that are not related to the meshing process and leaving just one in the damaged data), the most notable fact is that this result is achieved by using different SK technology parameters. The SK time resolutions for the highest probability of correct diagnosis are wildly different, while the SK threshold required still shows some variation.

\subsection{Effectiveness of damage diagnosis}

The non-adapted baseline set of processing parameters used in this study (SK resolution of mesh frequency and SK threshold of 0.2 ) results in percentages of correct diagnosis of $53 \%$ and $52 \%$, respectively, for the two speed and load combinations. In the low speed and load condition (Figure 7) it can be seen that this is because the gearbox almost always results in damage being diagnosed. The damaged cases would therefore mostly be diagnosed correctly, but the undamaged data would rarely be identified as such. The non-adapted parameters perform similarly for the high-speed, high-load case (Figure 8).

Adaptation of the SK technology parameters enables the probability of correct diagnosis to increase to $87 \%$ and $97 \%$ for low-speed, low-load and high-speed, high-load cases, respectively. However, the notable difference is that the optimised SK resolution and SK threshold values are different in each case. SK resolution values tested ranged from $0.25 \times$ fmesh to $4 \times$ fmesh and SK threshold values of 0.01 to 1 .

In the low speed and load scenario $(1500 \mathrm{r} / \mathrm{min}, 250 \mathrm{Nm})$ the highest probability of correct diagnosis was achieved when the SK resolution was $200 \mathrm{~Hz}$ and the SK threshold set at 1 (Figure 9).

Conversely, in the high-speed and high-load case $(3000 \mathrm{r} / \mathrm{min}$,

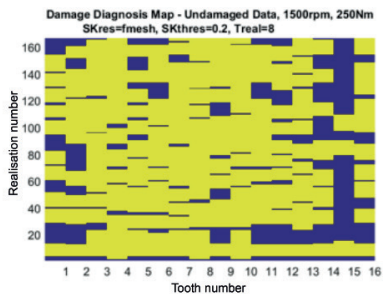

(a)

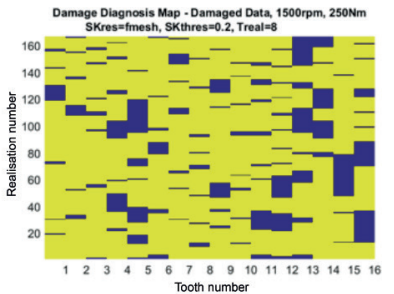

(b)
Figure 7. Damage diagnosis maps for $1500 \mathrm{r} / \mathrm{min}, 250 \mathrm{Nm}$ data when processed with the baseline parameters. The resultant probability of correct diagnosis is 53\%: (a) automated diagnosis undamaged; (b) automated diagnosis - damaged

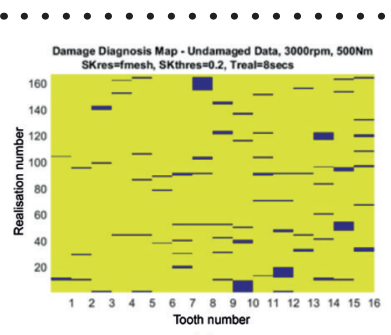

(a)

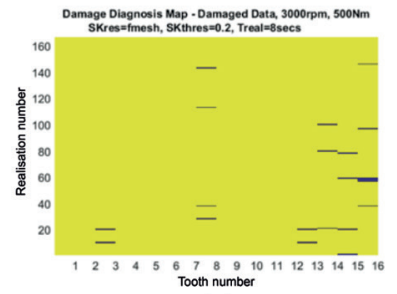

(b)
Figure 8. Damage diagnosis maps for $3000 \mathrm{r} / \mathrm{min}, 500 \mathrm{Nm}$ data when processed with the baseline parameters. The resultant probability of correct diagnosis is 52\%: (a) automated diagnosis undamaged; (b) automated diagnosis - damaged

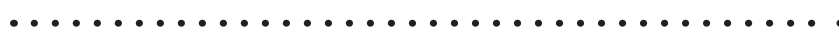
$500 \mathrm{Nm}$ ) the highest probability of correct diagnosis was at an SK resolution of $1200 \mathrm{~Hz}$ and an SK threshold of 0.75 (Figure 10).

Whilst this was not an exhaustive study of all possible parameters, it shows that even minor adjustment to the SK technology parameters can achieve large gains in damage diagnosis. There are some small signal elements in the undamaged data that

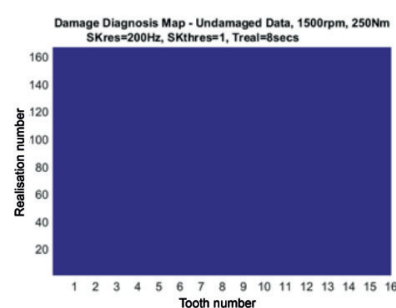

(a)

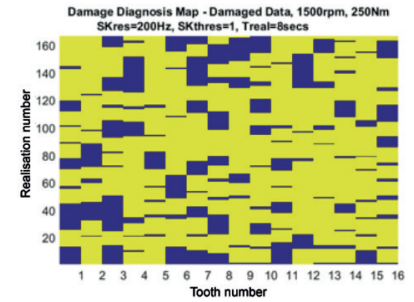

(b)
Figure 9. Damage diagnosis maps for $1500 \mathrm{r} / \mathrm{min}, 250 \mathrm{Nm}$ data when processed with an SK resolution of $200 \mathrm{~Hz}$ and SK threshold of 1 . The resultant probability of correct diagnosis is 87\%: (a) automated diagnosis - undamaged; (b) automated diagnosis - damaged

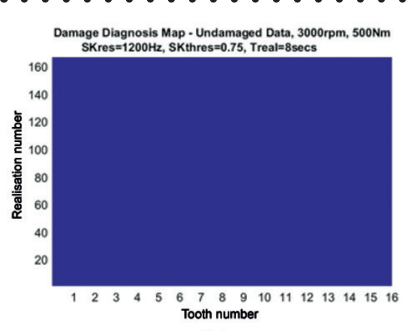

(a)

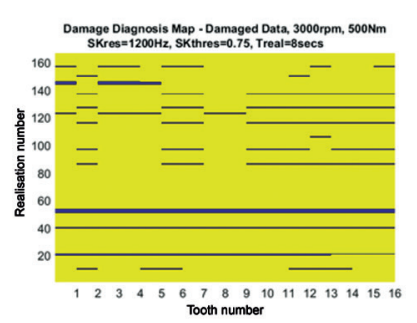

(b)
Figure 10. Damage diagnosis maps for $3000 \mathrm{r} / \mathrm{min}, 500 \mathrm{Nm}$ data when processed with an SK resolution of $1200 \mathrm{~Hz}$ and SK threshold of 0.75 . The resultant probability of correct diagnosis is $97 \%$ : (a) automated diagnosis - undamaged; (b) automated diagnosis - damaged 
are not related to gear meshing but still result in non-zero SK values. However, despite these signal abnormalities, the parameter adaptation has significantly improved the total probability of correct diagnosis in the two demonstrated cases.

Both sets of data used the same values within the automated decision-making codes, but it is also proposed that these could be optimised to each speed and load case to further improve the results. Initial observations suggest that the values used in this case were overly sensitive (based on the non-optimised diagnosis), but by adapting the SK values appropriately the effect of incorrectly chosen decision-making parameters is dramatically reduced. Seeing as these variables are also typically chosen through trial and error, this is a key observation for future work as less time will be required to find suitable decision-making variable values. Adapting the decision-making parameters to the speed and load conditions may also affect the optimal SK technology values as the parameters should ideally all be adapted together.

\section{Conclusions}

This paper has proposed and investigated the adaptation of the powerful spectral kurtosis signal processing techniques to the main operating parameters of rotating machinery, ie the effect that operating parameters have on the SK of gearbox vibration data. Signals were obtained from the same gearbox run in different combinations of speed and load, after which TSA and removal of mesh harmonics was performed. The SK calculation is performed based on the STFT of the local signal power, as is common throughout literature in the field.

It has been shown that optimisation of both the SK resolution and SK threshold are dependent on gearbox operating conditions. Adapting the SK technology parameters leads to an SK-based Wiener filter customised to the specific speed and load case, improving the consistency of the signal across each realisation, removing noise or other signal irregularities and resulting in increasing the probability of a correct diagnosis.

Correctly adapting the SK technology parameters allows damage to be correctly diagnosed even if the separation between damaged and undamaged data is minimal or data quality is compromised. The effectiveness of diagnosis improves if there are frequency bands with consistently high SK values in the data.

A baseline case was taken whereby four data files (one damaged and one undamaged for two combinations of speed and load) were processed using selected SK technology variables, after which the effect of adapting the SK resolution and SK threshold were demonstrated. Adapting each parameter achieved an increase in the probability of correct diagnosis from $53 \%$ to $87 \%$ and $52 \%$ to $97 \%$ for the two combinations of operating conditions. Thus, the proposed adaptation improved the effectiveness of gearbox diagnosis in conditions of varying speed and load, with effectiveness gains of 3.6 times and 16 times, respectively, in terms of total error probabilities.

The proposed adaptation is highly important for the diagnosis of gearboxes operating in non-stationary conditions (for example in the aerospace, marine, water, robotics and automotive industries).

\section{Acknowledgements}

The authors thank colleagues Dr H Nedunuri and Dr I Petrunin (both from Cranfield University) for providing initial sample sets of processing codes along with advice when required.

\section{References}

1. P D McFadden, 'Examination of a technique for the early detection of failure in gears by signal processing of the time domain average of the meshing vibration', Mechanical Systems and Signal Processing, Vol 1, No 2, pp 173-183, 1987.

2. $\mathrm{PD}$ McFadden, 'Determining the location of a fatigue crack in a gear from the phase of the change in the meshing vibration', Mechanical Systems and Signal Processing, Vol 2, No 4, pp 403-409, 1988.

3. M El Badaoui, V Cahouet, F Guillet, J Danière and P Velex, 'Modelling and detection of localised tooth defects in geared systems', Journal of Mechanical Design, Vol 123, No 3, pp 422430, 2001.

4. F Bonnardot, M El Badaoui, R B Randall, J Danière and F Guillet, 'Use of the acceleration signal of a gearbox in order to perform angular resampling (with limited speed fluctuation)', Mechanical Systems and Signal Processing, Vol 19, No 4, pp 766-785, 2005.

5. F Combet and L Gelman, 'An automated methodology for performing time synchronous averaging of a gearbox signal without speed sensor', Mechanical Systems and Signal Processing, Vol 21, No 6, pp 2590-2606, 2007.

6. N Sawalhi, R B Randall and H Endo, 'The enhancement of fault detection and diagnosis in rolling element bearings using minimum entropy deconvolution combined with spectral kurtosis', Mechanical Systems and Signal Processing, Vol 21, No 6, pp 2616-2633, August 2007.

7. J Antoni and R B Randall, 'The spectral kurtosis: application to the vibratory surveillance and diagnostics of rotating machines', Mechanical Systems and Signal Processing, Vol 20, No 2, pp 308-331, February 2006.

8. F Combet and L Gelman, 'Optimal filtering of gear signals for early damage detection based on the spectral kurtosis', Mechanical Systems and Signal Processing, Vol 23, No 3, pp 652-668, 2009.

9. F Combet, L Gelman and G Lapayne, 'Novel detection of local tooth damage in gears by the wavelet bicoherence', Mechanical Systems and Signal Processing, Vol 26, No 1, pp 218-228, 2012.

10. L Gelman, B Murray, T H Patel and A Thomson, 'Novel decision-making technique for damage diagnosis', Insight: Non-Destructive Testing and Condition Monitoring, Vol 55, No 8, pp 428-432, 2013.

11. L Gelman and I Petrunin, 'Novel anomaly detection based on the nearest neighbours and sequential methods', In: 8th International Conference on Condition Monitoring and Machinery Failure Prevention Technologies, CM 2011/MFPT 2011.

12. R Zimroz, W Bartelmus, T Barszcz and J Urbanek, 'Diagnostics of bearings in the presence of strong operating conditions of nonstationarity: a procedure of load-dependent feature processing with application to wind turbine bearings', Mechanical Systems and Signal Processing, Vol 46, No 1, pp 16-27, 2014.

13. C Fakher, B Walter, Z Radoslaw, F Tahar and H Mohamed, 'Gearbox vibration signal amplitude and frequency modulation', Shock and Vibration, No 4, pp 635-652, 2012.

14. F Chaari, W Bartelmus, R Zimroz, T Fakhfakh and M Haddar, 'Effect of load shape in cyclic load variation on dynamic behaviour of a spur gear system, Key Engineering Materials, Vol 518, pp 119-126, 2012.

15. Y Zhan, V Makis and A K S Jardine, 'Adaptive state detection of gearboxes under varying load conditions based on parametric modelling, Mechanical Systems and Signal Processing, Vol 20, No 1, pp 188-221, 2006.

16. L Gelman, K C Gryllias, B Shaw and M Vaidhianathasamy, 'Condition monitoring of gearboxes', In: International Conference on Condition Monitoring and Machinery Failure Prevention Technologies, CM/MFPT 2012.

17. L Gelman, H Nedunuri et al, 'Novel gear diagnosis technique based on the spectral kurtosis', In: 23rd International Congress on Sound and Vibration, 2016. 
2017-08-01

Novel adaptation of the spectral kurtosis for vibration diagnosis of gearboxes in non-stationary conditions

Gelman, Len

British Institute of Non-destructive Testing

Gelman L, Kolbe S, Shaw B, Vaidhianathasamy M, Novel adaptation of the spectral kurtosis for vibration diagnosis of gearboxes in non-stationary conditions, Insight: Non-Destructive Testing \& Condition Monitoring, Vol. 59, Issue 8, August 2017, pp. 434-439

http://dx.doi.org/10.1784/insi.2017.59.8.434

Downloaded from Cranfield Library Services E-Repository 